\title{
Statistical Molecular Dynamics Study of (111) and (100) Ni Nanocontacts: Evidences of Pentagonal Nanowires
}

\author{
P. García-Mochales, ${ }^{1}$ R. Paredes, ${ }^{2,3}$ S. Peláez, ${ }^{3}$ and P. A. Serena ${ }^{3}$ \\ ${ }^{1}$ Departamento de Física de la Materia Condensada, Universidad Autónoma de Madrid, Cantoblanco, 28049 Madrid, Spain \\ ${ }^{2}$ Centro de Física, Instituto Venezolano de Investigaciones Científicas Altos de Pipe, 1020-A Caracas, Venezuela \\ ${ }^{3}$ Instituto de Ciencia de Materiales de Madrid, Consejo Superior de Investigaciones Científicas, c/ Sor Juana Inés de la Cruz 3 , \\ Cantoblanco, 28049 Madrid, Spain
}

Correspondence should be addressed to P. A. Serena, pedro.serena@icmm.csic.es

Received 21 September 2007; Accepted 11 January 2008

Recommended by Jun Lou

We present molecular dynamics calculations on the evolution of Ni nanowires stretched along the (111) and (100) directions, and at two different temperatures. Using a methodology similar to that required to build experimental conductance histograms, we construct minimum crosssection histograms $H\left(S_{m}\right)$. These histograms are useful to understand the type of favorable atomic configurations appearing during the nanowire breakage. We have found that minimum crosssection histograms obtained for (111) and (100) stretching directions are rather different. When the nanowire is stretched along the (111) direction, monomer and dimer-like configurations appear, giving rise to well-defined peaks in $H\left(S_{m}\right)$. On the contrary, (100) nanowire stretching presents a different breaking pattern. In particular, we have found, with high probability, the formation of staggered pentagonal nanowires, as it has been reported for other metallic species.

Copyright ( $) 2008$ P. García-Mochales et al. This is an open access article distributed under the Creative Commons Attribution License, which permits unrestricted use, distribution, and reproduction in any medium, provided the original work is properly cited.

\section{INTRODUCTION}

Nanotechnology involves the design, fabrication, and application of structures by controlling their composition, shape, and size at the nanometer scale [1]. In particular, the control of these properties will allow to exploit a whole set of novel physical and chemical features in future nanoelectronics development. Such development includes the study of the electron transport through different candidates to be used as nanoelectronics building blocks. Among these candidates, metallic nanowires or nanocontacts will play a relevant role $[2]$.

The interest on metallic nanowires and nanocontacts rises from their rich phenomenology. Electron transport through metallic nanowires present ballistic features and, in addition, well-defined electron transport modes or channels appear associated to the transversal confinement of electrons for those nanowires with diameters of the order of few Fermi wavelengths $\lambda_{F}$. In the ballistic limit, the conductance $G$ (inverse of the nanowire resistance $R, G=1 / R$ ) is described in terms of the transmission probabilities associated to these transport channels $[3,4]$.

Several experimental techniques have been used to form metallic nanocontacts and nanowires. Scanning tunneling microscopy (STM) [5-7] and mechanically controllable break junction (MCBJ) [8] methods are standard approaches to study the formation and rupture of nanocontacts under different experimental conditions. Metallic nanowires are also obtained using electron-beam irradiation inside ultrahigh vacuum (UHV) transmission electron microscopes (TEM) [9] or using electrochemical methods [10].

The electric characterization of a metallic nanowire is usually done during its rupture. This rupture is achieved using SPM or MCBJ methods, leading to the acquisition of a nanowire conductance trace $G(t)$. In order to obtain relevant information concerning the electronic transport through nanowires of a given metallic species, conductance histograms $H(G)$ are constructed by accumulating many different conductance traces. Usually, conductance histograms present well-defined peaked structures, reflecting 
the existence of preferred conductance values. Such conductance peaks are usually interpreted in terms of conductance quantization [8] or favorable atomic arrangements [11, 12].

The interpretation of a conductance histogram is a very difficult task, since it merges mechanical and electrical information as well as information coming from different conductance channels as it happens for polyvalent metals [13]. In spite of these interpretation problems, conductance histograms have become a standard tool to analyze the properties of metallic nanowires [2].

Electronic transport in atomic-sized magnetic nanowires has been profusely studied [14-32] since magnetic nanowires are a very interesting topic for future devices and applications. However, to the presence of a new degree of freedom, "spin" requires an additional effort to study and interpret experimental conductance histograms.

Among those studies dealing with magnetic nanowires, several works have addressed the study of nickel conductance histograms. A pioneering experiment (performed at RT and without applied magnetic field) showed that Ni conductance histograms constructed with thousands of unselected conductance traces presented a featureless structure [15]. However, very different results, showing well-defined peaks, were reported for $\mathrm{Ni}$ conductance histograms [16] obtained in UHV. In particular, conductance histograms presented clear evidences of fractional conductance quantization. Moreover, these experiments showed that conductance histograms depend on the applied magnetic field as well as on the temperature. A different set of experiments carried out at RT showed that conductance histograms (constructed with less than 100 conductance traces) suffered strong modifications when the applied magnetic increases $[17,29]$. Finally, experiments carried out at $4 \mathrm{~K}$, in UHV conditions, and using thousand of conductance traces showed $\mathrm{Ni}$ conductance histograms with two well-defined peaks around $\sim 1.6 G_{0}$ and $\sim 3.1 G_{0}$, respectively $[21,22,24,26,31]$. The position of both peaks was not modified under application of strong magnetic fields. The position of the first peak is consistent with previous experimental results [14] and may be explained in terms of monomer and dimer configurations appearing during the last stages of the nanowire breaking process $[14,33]$.

Encouraged by the existence of contradictory experimental results, we have focused the present study on the role played by the mechanical behavior during the breaking process of $\mathrm{Ni}$ nanowires at low and room temperatures. Both situations are well below the Ni melting temperature $\left(T_{m}=1728 \mathrm{~K}\right)$ and, in principle, a similar mechanical behavior is expected. However, we need to exclude this thermal effect as the origin of those marked differences between low temperature and room temperature $H(G)$. The aim of the present work is to carry out a statistical study of the structural evolution of $\mathrm{Ni}$ nanowires under stretching at low and room temperatures following a well tested methodology [12, 34-37]. We will analyze the role of the stretching direction studying two different cases: (111) and (100). An additional motivation for carrying out the present study is also related with the different computational histograms that have been recently reported for (111) and (100) orientations in Ni nanocontacts [31, 37, 38].

The paper is organized as follows: in the following section we describe the computational approach used to construct computational histograms. In Section 3, we present the results for two temperatures as well as two nanowire stretching directions; and, finally, Section 4 summarizes our main conclusions.

\section{COMPUTATIONAL METHODS}

The main goal of the present study is to understand the role of temperature and nanowire orientation on the nanowire evolution from a statistical perspective. We follow a similar strategy to the experimental one, simulating hundreds of independent breaking events, in order to determine whether the geometry presents some preferred configurations that give rise to well-defined peaked structures in the conductance histogram.

The simulation of metallic nanowire breaking events has been carried out using standard molecular dynamics (MD) methods based on a description of the atom-atom interaction based on the atomic electron densities. MD has been extensively used to study the structure and rupture of metallic nanowires. In the past years, metallic nanowire MD simulations focused on the description of single formation or breaking events, using different interatomic potentials but neglecting the study of statistical effects [6, 7, 39-49]. More recently, several $\mathrm{MD}$ studies on breaking and formation processes of nanocontacts have statistically analyzed the appearance of preferred atomic configurations in order to establish correlations with these peaks found in experimental conductance histograms at different temperatures $[12,31$, 34-38, 50]. Some of these statistical MD simulation studies $[36,38,50]$ have been carried out using a hybrid scheme, where MD configurations are used as starting point for the calculation of the electronic transport using more sophisticated quantum methods. These studies reveal that there is a complex relation between the atomic configuration and the conductance histograms.

We have simulated the nanowire dynamics using an MD scheme where atomic interaction is represented by embedded atom method (EAM) potentials [51, 52]. In EAM, the potential energy function for the system reads

$$
E=\frac{1}{2} \sum_{i j} \phi\left(r_{i j}\right)+\sum_{i} F\left(\bar{\rho}_{i}\right),
$$

where $i$ and $j$ run over the number of atoms. In the first term, $\phi(r)$ corresponds to a pair potential depending only on the distance $r_{i j}$ between every pair of "different" atoms $i$ and $j$. The second term is the so-called embedding energy, which depends on the mean electronic density $\bar{\rho}_{i}$ at atom $i$ 's location. This density is approximated in EAM as the sum of the contributions due to the surrounding atoms, $\bar{\rho}_{i}=$ $\sum_{j \neq i} \rho\left(r_{i j}\right)$. Then, the embedding energy is calculated by evaluating and summing the embedding function $F(\rho)$ at each atom's position. Depending on the material and the specific physical properties to be studied, different pair potential 


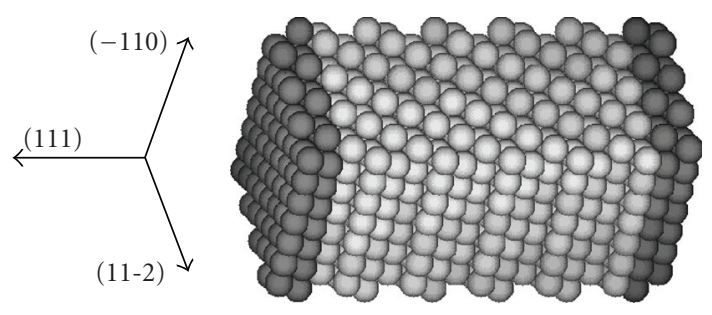

(a)

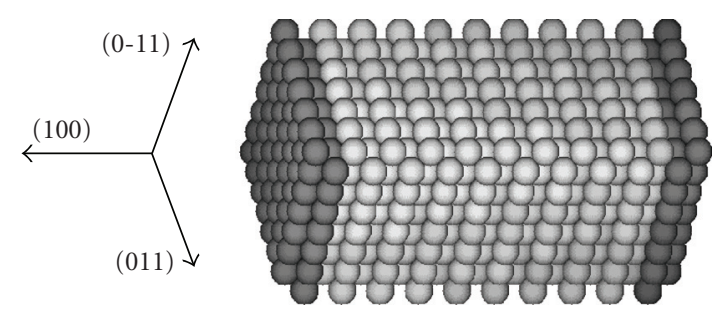

(b)

Figure 1: Initial configurations of (a) (111) and (b) (100) Ni nanowires.

$\phi\left(r_{i j}\right)$, embedding $F(\rho)$, and density $\rho\left(r_{i j}\right)$ functions can be defined. In the present study, we have used the EAM parameterization proposed by Mishin et al. [53]. This parameterization has been constructed by fitting almost 30 different properties obtained from experimental measurements or ab-initio calculations. These fitted properties correspond to bulk as well as surface properties, therefore taking into account low-dimensional configurations as those found during the last nanowire breaking stages.

Nanowire dynamics have been studied at constant temperature $T$ using a standard velocities scaling algorithm at every MD step. Two temperatures $(4 \mathrm{~K}$ and $300 \mathrm{~K}$ ) have been considered in this work in order to describe the $\mathrm{Ni}$ nanowire dynamics at low and room temperatures. The time interval of an MD step is $\delta t=10^{-2} \mathrm{ps}$. Atomic trajectories and velocities were determined using conventional Verlet velocity integration algorithms. We have checked that the obtained minimum cross-section time evolution curves and histograms obtained with this time step are very similar to that obtained with a shorter time interval.

The simulation of a single nanowire breaking event consists of three stages. The first stage corresponds to the definition of the initial unrelaxed structure. We consider a bulk super-cell with parallelepiped shape, containing hundreds of atoms ordered according to an fcc structure with bulk Ni lattice parameter $(a=3.52 \AA)$. The initial parallelepiped height will correspond to the stretching direction and is larger than the base edges. We define the $z$ axis as the stretching (pulling) direction. In the present study we have considered two different stretching directions: (111) and (100). For the (111) case we have used an initial nanowire formed by 18 layers containing 56 atoms each (i.e., a total of 1008 atoms). For the (100) case we have used a nanowire of 21 layers with 49 atoms per layer (i.e., 1029 atoms). Figure 1 shows the two initial configurations corresponding to (111) and (100) nanowires. At the beginning of the simulation, the velocity of each atom is assigned at random according to the Maxwellian distribution that correspond to the simulation temperature.

The second stage corresponds to the relaxation of the bulk-like initial structure. Firstly, we define two supporting bilayers at the top and bottom of the supercell. Atomic $x$ and $y$ coordinates within these bilayers will be kept frozen during the simulation. The nanowire will remain attached to these two bulk-like supporting bilayers during the relaxation stage. This stage lasts for $3000 \mathrm{MD}$ steps in order to optimize the geometry of the isolated parallelepiped-like nanowire. Notice that the presence of both supporting frozen bilayers also avoids the appearance of phase transformation upon loading as it has been noticed for narrow Ni nanowires without such supporting bilayers [54].

During the third stage (stretching process), the $z$ coordinate of those atoms forming the top (down) frozen bilayer is forced to increase (decrease) a quantity $\Delta L_{z}=10^{-4} \AA$ after every MD step. This incremental process simulates the separation of the supporting bilayers in opposite directions at constant velocity of $2 \mathrm{~m} / \mathrm{s}$, giving rise to the subsequent nanowire fracture. The rest of the atoms move following the forces derived from their EAM-like interaction with the surrounding atoms. Notice that the stretching velocity is much larger than that used in experiments. However, our computational description of the nanowire breaking is comparable to that of actual experimental traces since the stretching velocity is smaller than the sound speed in nickel.

During the stretching stage, the accurate knowledge of the atomic coordinates and velocities allows the determination of the minimum cross-section $S_{m}$. This quantity provides information on the favorable configurations appearing during the nanowire evolution under stretching. Furthermore, $S_{m}$ provides a first-order approximation of the conductance $G[55,56]$.

The minimum cross-section $S_{m}$ is calculated in units of atoms following standard procedures that have been successfully used in previous studies [41, 42]. In our case, we define the radius $r_{0}$ to be equal to half the fcc (111) interplanar distance $\left(r_{0}=d_{111} / 2\right)$. We assign a volume $V_{0}=4 \pi r_{0}^{3} / 3$ to each atom. In order to calculate the crosssection $S_{i}$ at a given $z_{i}$ position, we firstly compute the total atomic volume $V_{\text {tot, } i}$ inside a "detecting cursor" width $\Delta z$. We have used $\Delta z=d_{111}$. The quantity $S_{i}=V_{\text {tot }, i} / V_{0}$ corresponds to the nanowire section (in number of atoms) at the $z_{i}$ position. The detecting cursor moves along the $z$ axis between the two frozen bilayers, using a step equal to $0.1 \times d_{111}$. This allows to calculate the cross-section $S_{i}$ along the nanowire. Finally, from the set of collected $S_{i}$ values, we determine the minimum cross-section value $S_{m}$. Note that the cursor size $\Delta z$ is kept fixed independently on the nanowire crystalline direction along the $z$ axis. This allows a true comparison between histograms obtained for different orientations, especially at the last breaking stages. In our study $S_{m}$ is calculated every $10 \mathrm{MD}$ steps. We consider that the nanowire breaking process is completed when $S_{m}=0$. 


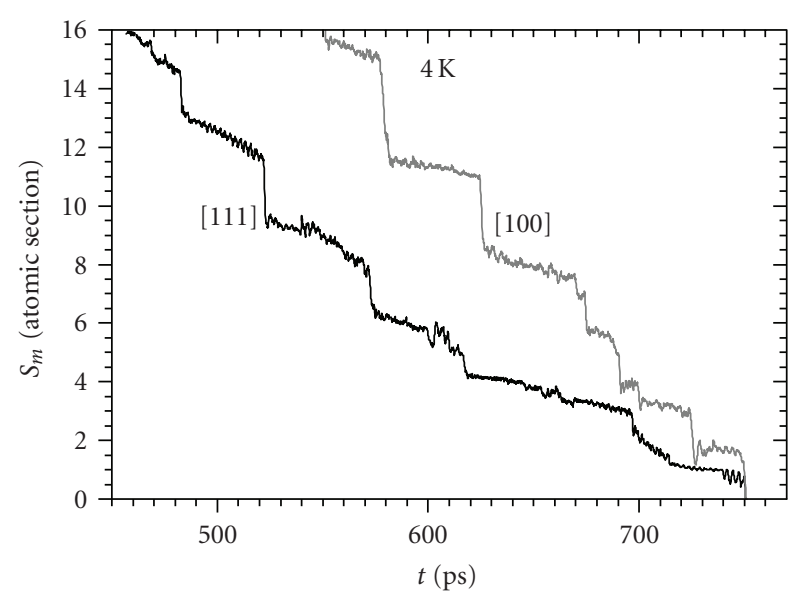

(a)

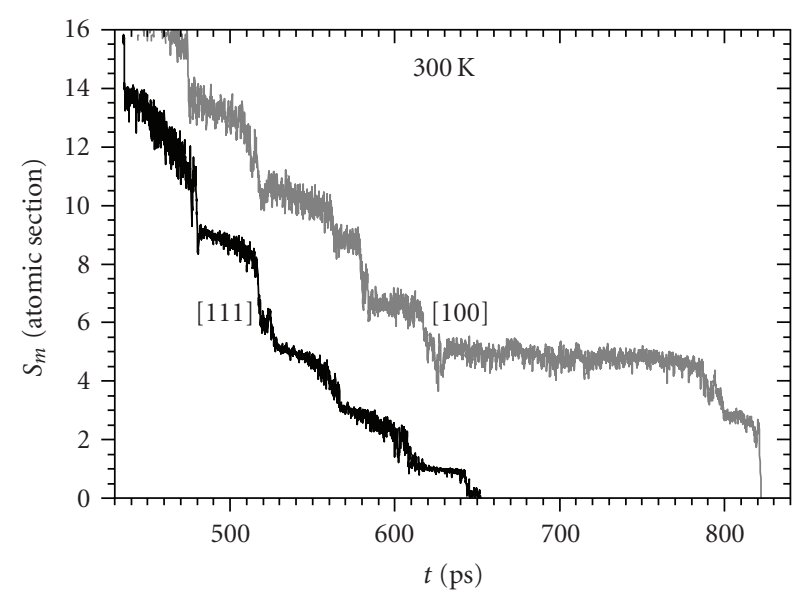

(b)

Figure 2: Minimum cross-section $S_{m}$ evolution of simulated breakage processes of Ni nanowires along the (111) (black curves) and (100) (grey curves) stretching directions at $4 \mathrm{~K}$ (a) and $300 \mathrm{~K}$ (b).

\section{RESULTS AND DISCUSSION}

The evolution of $S_{m}$ versus time shows a typical staircase trace. In Figure 2 we show four examples of $S_{m}(t)$ that illustrate this behaviour. $S_{m}(t)$ traces show a stepped profile with well-marked jumps associated to atomic rearrangements occurring at the nanowire. We have verified that these jumps are correlated with jumps in the force acting on the supporting slabs. In general, $S_{m}$ monotonically decreases between two subsequent jumps reflecting the existence of elastic stages. These elastic stages have been associated to the experimentally observed conductance plateaus. As it is expected, $S_{m}$ presents at RT larger fluctuations than at $4 \mathrm{~K}$. This has been observed for both stretching orientations.

In order to visualize the possible differences between low and room temperatures and between (111) and (100) stretching directions, we have depicted in Figure 3 five atomic configurations for each trace shown in Figure 2. Although it is very difficult to make comparisons among those structural evolutions, we can see that, in general, the nanowires break-forming three types of configurations at the last stage of the breaking process: monomers, dimers, and others. The monomer structure is characterized by a central atom standing between two pyramid-like structures. This configuration usually shows a plateau around $S_{m} \sim 1$. In the dimer structure, the apex atoms of two opposite pyramidal configurations form a diatomic chain. The third type of final configurations presents more complex structures. In these cases we generally observe an abrupt jump from $S_{m} \geq 2$ (i.e., structures formed by two or more atoms) to $S_{m}=0$. As expected [45], we have not found the presence of long atomic chains (formed by three or more atoms).

In our study we have found that monomer and dimer structures usually appear for nanowires stretched along (111) direction. On the contrary, more complex structures (i.e., not classifiable as monomers or dimers) appear with higher probability at the latest stages of nanowires strained along the (100) stretching directions.
The minimum cross-section histograms $H\left(S_{m}\right)$ have been built by accumulating $S_{m}(t)$ traces acquired during the simulation of hundreds of nanowire stretching processes. In Figure 4 we show the histograms $H\left(S_{m}\right)$ for $4 \mathrm{~K}$ and $300 \mathrm{~K}$, constructed with 300 independent breakages for the (111) and (100) stretching directions. The different histograms are depicted in the range $0<S_{m}<12$. A first inspection of these figures reveals the existence of well-defined peaks associated to preferred nanowire configurations as it has been shown in previous works [12, 34-37].

The $H\left(S_{m}\right)$ histograms associated to the (111) stretching direction present a well-defined peaked structure at low and room temperature. The main difference we have found corresponds to the small increase of the peak located at $S_{m} \sim 5$ at RT. For the (100) case, the low-temperature histogram presents a noisy structure whereas at $T=300 \mathrm{~K}$ the histogram peaks show rounded shapes and the general structure has less noise. Small peaks at $T=4 \mathrm{~K}$ correspond to metastable configurations with slightly higher cohesive energies with respect to other metastable configurations. The increase of temperature allows the exploration of more configurations during the stretching process, and, in this way, those metastable configurations with local minimum energy are easily accessible, leading to a better definition of their associated $H\left(S_{m}\right)$ peaks. In addition, the (100) $H\left(S_{m}\right)$ histogram presents a well-marked $S_{m}=5$ peak at $T=300 \mathrm{~K}$. We will discuss later the origin of this protruding peak which is not developed at low temperatures.

Our main finding is that $H\left(S_{m}\right)$ histograms are very dependent on the stretching (i.e., the nanowire axis) direction. Whereas the (111) direction provides histograms with well-defined decreasing peaked structure in the low $S_{m}$ region, the situation dramatically changes when we consider the (100) direction. The later case presents $H\left(S_{m}\right)$ histograms with small peaks in the region $S_{m}<2$. These differences between (111) and (100) stretching directions are consistent with previously published results $[37,38]$. 

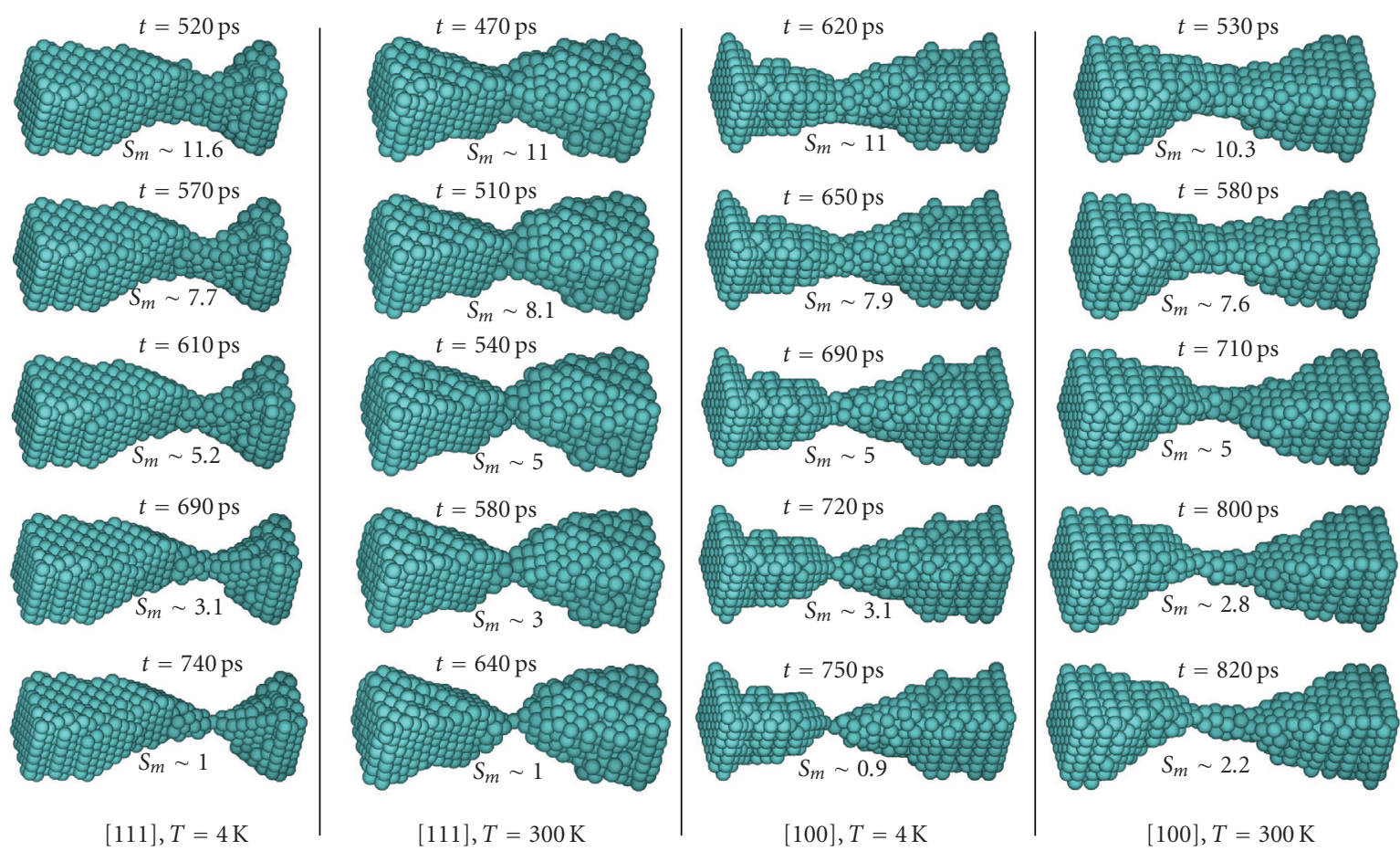

FIGURE 3: Snapshots at different evolution times of those nanowires whose $S_{m}(t)$ traces were shown in Figure 2. Each column corresponds to a given combination of the nanowire stretching direction $((111)$ or $(100))$ and temperature $(T=4 \mathrm{~K}$ or $T=300 \mathrm{~K})$. Each snapshot includes information on the simulation time and the minimum cross-section $S_{m}$ at that time.

We have pointed out that the peak located at $S_{m} \sim 5$ of the histograms $H\left(S_{m}\right)$ increases when the temperature goes from $T=4 \mathrm{~K}$ to RT. This increase is small for the (111) case. The situation is rather different for the (100) case, noticing that this peak increases a factor of 2 approximately (see Figure 4). The MD simulation approach allows to monitor the evolution of the full set of atomic coordinates during the breaking process. In order to know with more detail the origin of the $S_{m}=5$ peak appearing in the (100) case, we have depicted in Figure 5 a typical nanowire stretched along the (100) direction. We have chosen a snapshot corresponding to the $4.5<S_{m}<5.5$ region. It is clear that the origin of the huge peak is related to the tendency of the system to form long wires with $S_{m} \sim 5$. In general, this type of nanowires beaks without a progressive diminishing of its atomic section, that is, the system does not form monomers or dimers just before the rupture. At this point we should mention that these long structures are very common for the (100) stretching direction. However, these long rod-like structures appear seldom in the (111). This smaller probability agrees with the slight increase detected for the $H\left(S_{m}=5\right)$ peak when $T$ increases.

A closer inspection to Figure 5 allows to determine the atomic structure of these long wires. We have found that these wires present a well-defined sequence $\cdots-5-1-5-$ $1-\cdots$. This sequence does not correspond to any crystallographic fcc or bcc structure. This type of arrangement is not seen at $4 \mathrm{~K}$ because a larger temperature is required to explore and overcome those energy barriers leading to configurations able to develop these pentagonal chains. In addition, we can easily see that these pentagonal nanowires are formed by subsequent-staggered parallel pentagonal rings (with a relative rotation of $\pi / 5$ ) connected with single atoms. A similar, but shorter, structure was found for $\mathrm{Cu}$ (111) breaking nanowires using MD simulations [57]. The stability of such pentagonal $\mathrm{Cu}$ nanowires was later confirmed by ab-initio calculations [58], demonstrating that staggered pentagonal nanowires are favorable configurations. More recently, $\mathrm{MD}$ tight-binding calculations have determined the presence of such pentagonal -5-1-5-1- nanowires during the breaking process [59] of (110) $\mathrm{Cu}$ nanowires although these pentagonal patterns were not frequently observed for nanowires along (100) and (111) directions. Pentagonal motives also appear in infinite $\mathrm{Al}$ nanowires [60]. Therefore, it seems that the formation in MD simulation of staggered pentagonal $\mathrm{Ni}$ nanowires follows the trend observed for other metallic species.

We have checked that formation of staggered pentagonal nanowires is favorable within the EAM scheme using the Mishin et al. potential. In fact, we have found from a set of conjugate gradients geometry minimization procedures that staggered nanowires present a cohesive energy of $3.66 \mathrm{eV}$ per atom whereas the cohesive energy of the nonstaggered configuration (without the relative rotation of $\pi / 5$ ) is $3.40 \mathrm{eV}$. The optimized geometry of the staggered pentagonal $\mathrm{Ni}$ nanowire presents two subsequent parallel pentagonal rings separated each other by a distance $d_{5-5}=2.22 \AA$. The optimized pentagonal ring side takes the value $l_{5}=2.55 \AA$. The geometry parameters we have found for those pentagonal nanowires obtained from the stretching procedure present 


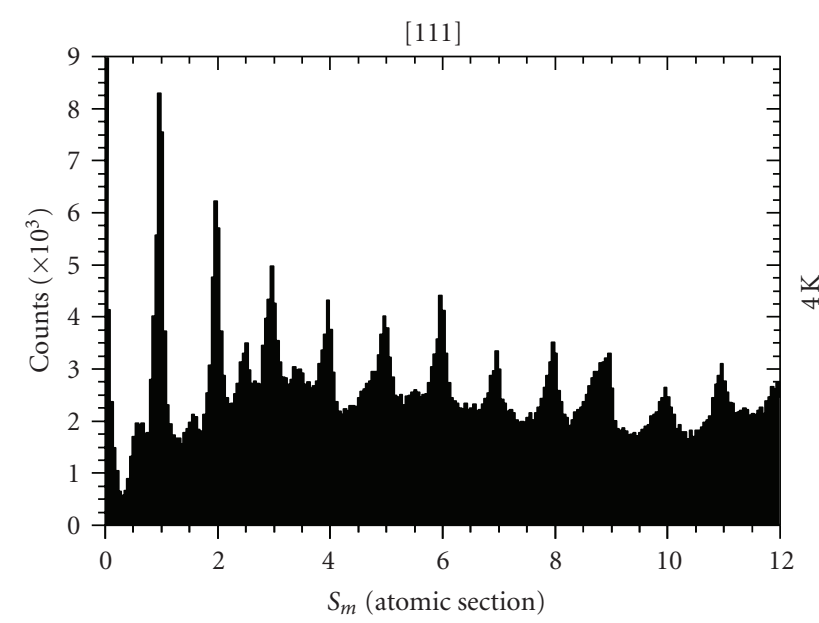

(a)

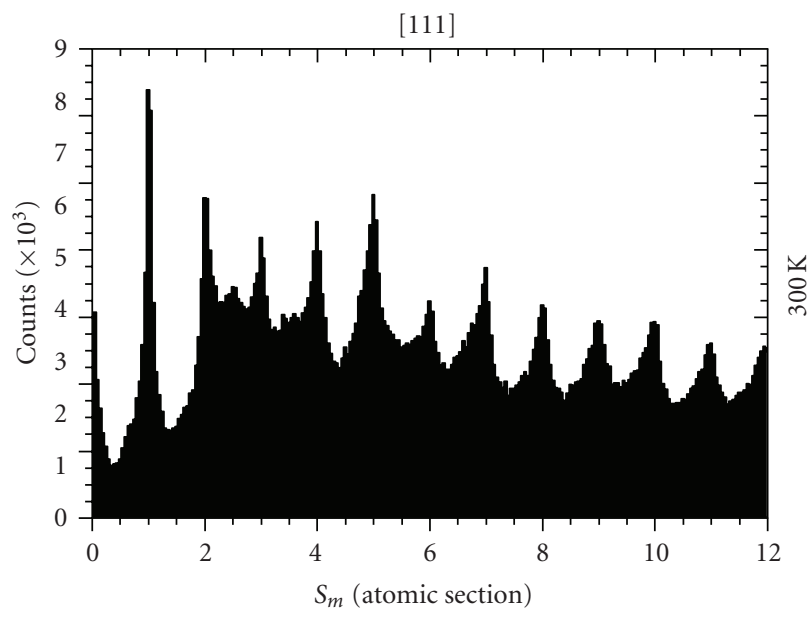

(c)

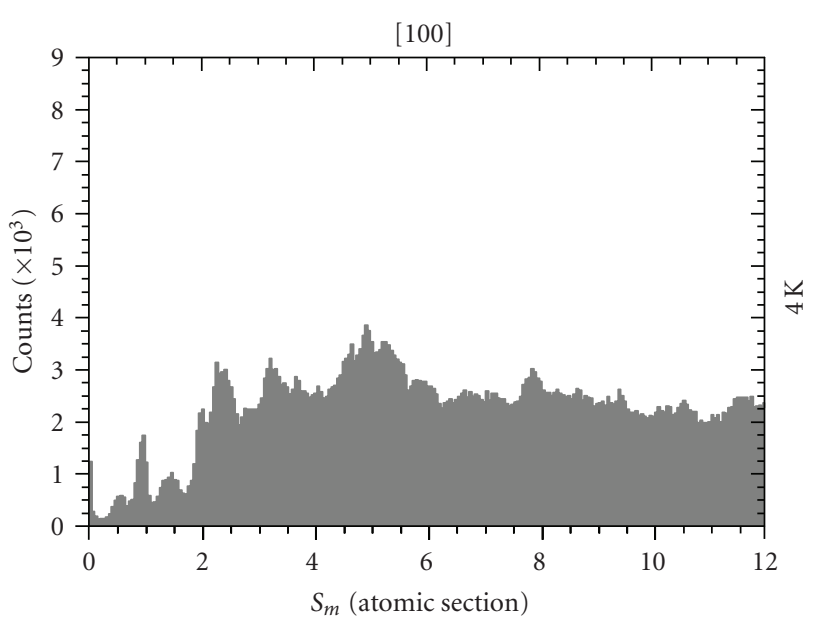

(b)

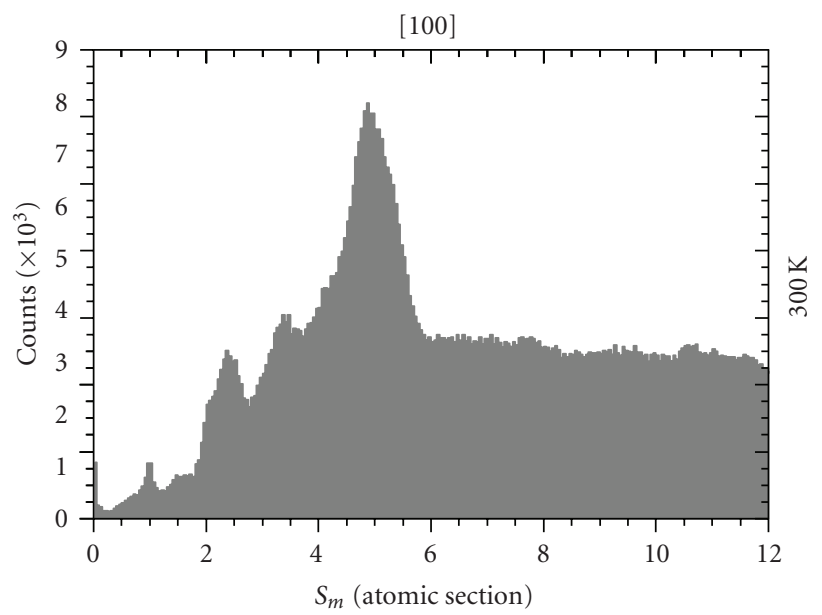

(d)

FIGURE 4: Minimum cross-section $S_{m}$ histograms built with 300 independent Ni nanowire ruptures under stretching along the (111) (a,c) and (100) (b,d) directions at $T=4 \mathrm{~K}(\mathrm{a}, \mathrm{b})$ and $300 \mathrm{~K}(\mathrm{c}, \mathrm{d})$.

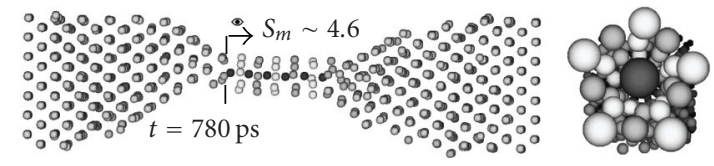

FIGURE 5: Longitudinal (left) and cross-section (right) views of a nanowire stretched along the (100) direction. The snapshot was acquired for an atomic configuration with minimum cross-section close to $S_{m} \sim 4$.6. The cross-section image shows a perspective view of the nanowire as seen from the position indicated on the longitudinal view. This image illustrates the appearance of staggered pentagonal structures $-5-1-5-1-$.

values of $d_{5-5}$ and $l_{5}$ very similar to those obtained for the free pentagonal nanowire. We have also found, using MD simulations, that the pentagonal nanowire structure is rather stable in the temperature range 3-300 K.

Therefore, it seems that the nanowire stretching along the (100) direction provides some mechanisms that allow to reach this favorable structures. In some sense, the stretching- induced formation of pentagonal nanowires is very similar to the formation of linear atomic chains (LACs) where single atoms are consecutively incorporated into the LAC from the stretched nanowire before its final breakage [2]. However, the formation of pentagonal chains involves units of 6 atoms $(1+5$ structures $)$ and a full description of the underlying mechanical process is a very complicated task that will be afforded in a deeper study.

\section{CONCLUSIONS}

We have carried out hundreds of MD simulations of $\mathrm{Ni}$ nanowire breaking processes using the EAM approach to describe the interatomic many body interaction. From these simulations we are able to follow the evolution of the nanowire minimum cross-section $S_{m}(t)$. By adding hundreds of $S_{m}(t)$ traces, we have constructed computational minimum cross-section histograms $H\left(S_{m}\right)$ that statistically unveil the presence of preferred configuration during the elongation and breaking history. The last stages of the nanowire 
breaking process are of special interest since electron transport is determined by a cross section formed by few atoms. We have found that monomers, dimers, and other more complex structures are present at the latest stages of the breaking events. We did not find linear atomic chains of three or more atoms for all the systems and stretching directions we analyzed.

We have found that $H\left(S_{m}\right)$ histograms do not depend dramatically on the temperature within the analyzed temperature range (below $300 \mathrm{~K}$ ). In general, we have only noticed rounding of the peaked structure of $H\left(S_{m}\right)$, the suppression of small $S_{m}$ fluctuations, and the increase of the peak located at $S_{m}=5$. This increase is larger for the (100) case. The absence of large temperature effects is consistent with the fact that bulk Ni melting temperature is rather large $(\approx 1728 \mathrm{~K})$, and then RT is not enough to activate additional mechanisms as surface diffusion effects that could modify the nanowire breaking dynamics.

During stretching along the (111) direction, the system tends to form a bipyramid structure that forms monomers and dimers at the narrowest nanocontact region. These monomers and dimers give rise to the $S_{m} \sim 1$ peak appearing in $H\left(S_{m}\right)$.

For the (100) stretching direction, $H\left(S_{m}\right)$ does not present large peaks in the low $S_{m}$ region $\left(S_{m}<2\right)$, thus indicating that monomers and dimers appear with less probability than for the (111) situation. The nanowire is deformed under stretching forming elongated (rod-like) structures. The formation of these long structures increases when temperature increases from $4 \mathrm{~K}$ to RT. At RT we found a dramatic increase of those peaks located at $S_{m} \sim 5$. We have confirmed that this peak is caused by the presence of long staggered pentagonal chains with $\cdots-5-1-$ 5-1 $\cdots$ structures. These pentagonal nanowires were also occasionally found for the (111) case (giving rise to the increase of the peak $\left.H\left(S_{m} \sim 5\right)\right)$. In relation with the formation of long chains in MD simulations, a recent work [61] points towards the higher ductibility predicted by EAM potentials in comparison with other interatomic potentials, leading to the formation of long structures. However, more detailed statistical analysis is required in order to know the actual effects of the chosen potential on the formation of elongated structures (including the pentagonal ones we have described).

In spite of our model limitations to perform the comparison with experiments, we can extract few key ideas from the present MD simulations. (i) The presence of well-marked $H\left(S_{m}\right)$ peaks is consistent with the appearance of preferred conductance values in the conductance histogram. (ii) The behavior of $H\left(S_{m}\right)$ at $4 \mathrm{~K}$ is rather similar to that noticed at $T=300 \mathrm{~K}$, and this implies that mechanical aspects do not explain differences between low and room temperature experimental conductance histograms. (iii) The first broad conductance peak appearing in experiments is consistent with the presence of monomers and dimers appearing during the last stages of the breaking process associated to (111) stretching direction. (iv) We have detected the formation of long pentagonal nanowires at RT for the (100) stretching direction. These structures contribute to the formation of a large $S_{m}=5$ peak. However, this large peak has not been observed in RT experimental conductance histograms. At this point we must be cautious when interpreting experimental conductance histograms, specially at RT, since the complex behavior of $\mathrm{Ni}$ conductance histograms may be attributed to chemiadsorbed atoms on the nanowire [26] or to ballistic magnetoresistence (BMR) effects $[19,20]$.

\section{ACKNOWLEDGMENTS}

The authors thank J. L. Costa-Krämer, M. Díaz, J. J. Palacios, C. Guerrero, E. Medina, and A. Hasmy for the helpful discussions. This work has been partially supported by the CSIC-IVIC researchers exchange program and the Spanish DGICyT (MEC) through Projects FIS2005-05137, BFM2003-01167-FISI, and FIS2006-11170-C02-01, and by Madrid Regional Government through the Programs S0505/MAT/0303 (NanoObjetos-CM) and S-0505/TIC/0191 (Microseres-CM). R.Paredes acknowledges the Spanish MEC by the financial support through its Researchers Exchange and Mobility Programme. Mochales also acknowledges Spanish MEC by its financial supprot through the RyC Programme.

\section{REFERENCES}

[1] C. P. Poole and F. J. Owens, Introduction to the Nanotechnology, Wiley-VCH, Weinheim, Germany, 2003.

[2] N. Agraï, A. L. Yeyati, and J. M. van Ruitenbeek, "Quantum properties of atomic-sized conductors," Physics Reports, vol. 377, no. 2-3, pp. 81-279, 2003.

[3] R. Landauer, "Electrical resistance of disordered one-dimensional lattices," Philosophical Magazine, vol. 21, no. 172, pp. 863-867, 1970.

[4] R. Landauer, "Electrical transport in open and closed systems," Zeitschrift für Physik B, vol. 68, no. 2-3, pp. 217-228, 1987.

[5] J. I. Pascual, J. Méndez, J. Gómez-Herrero, A. M. Baró, N. García, and V. T. Binh, "Quantum contact in gold nanostructures by scanning tunneling microscopy," Physical Review Letters, vol. 71, no. 12, pp. 1852-1855, 1993.

[6] L. Olesen, E. Laegsgaard, I. Stensgaard, et al., "Quantized conductance in an atom-sized point contact," Physical Review Letters, vol. 72, no. 14, pp. 2251-2254, 1994.

[7] L. Olesen, E. Laegsgaard, I. Stensgaard, et al., "Olesen et al. reply:" Physical Review Letters, vol. 74, no. 11, p. 2147, 1995.

[8] J. M. Krans, J. M. van Ruitenbeek, V. V. Fisun, I. K. Yanson, and L. J. de Jongh, "The signature of conductance quantization in metallic point contacts," Nature, vol. 375, no. 6534, pp. 767769, 1995.

[9] Y. Kondo and K. Takayanagi, "Gold nanobridge stabilized by surface structure," Physical Review Letters, vol. 79, no. 18, pp. 3455-3458, 1997.

[10] C. Z. Li and N. J. Tao, "Quantum transport in metallic nanowires fabricated by electrochemical deposition/ dissolution," Applied Physics Letters, vol. 72, no. 8, pp. 894896, 1998.

[11] A. I. Yanson and J. M. van Ruitenbeek, "Do histograms constitute a proof for conductance quantization?" Physical Review Letters, vol. 79, no. 11, p. 2157, 1997. 
[12] A. Hasmy, E. Medina, and P. A. Serena, "From favorable atomic configurations to supershell structures: a new interpretation of conductance histograms," Physical Review Letters, vol. 86, no. 24, pp. 5574-5577, 2001.

[13] E. Scheer, P. Joyez, D. Esteve, C. Urbina, and M. H. Devoret, "Conduction channel transmissions of atomic-size aluminum contacts," Physical Review Letters, vol. 78, no. 18, pp. 35353538, 1997.

[14] C. Sirvent, J. G. Rodrigo, S. Vieira, L. Jurczyszyn, N. Mingo, and F. Flores, "Conductance step for a single-atom contact in the scanning tunneling microscope: Noble and transition metals," Physical Review B, vol. 53, no. 23, pp. 16086-16090, 1996.

[15] J. L. Costa-Krämer, "Conductance quantization at room temperature in magnetic and nonmagnetic metallic nanowires," Physical Review B, vol. 55, no. 8, pp. R4875-R4878, 1997.

[16] H. Oshima and K. Miyano, "Spin-dependent conductance quantization in nickel point contacts," Applied Physics Letters, vol. 73, no. 15, pp. 2203-2205, 1998.

[17] T. Ono, Y. Ooka, H. Miyajima, and Y. Otani, " $2 e^{2} / h$ to $e^{2} / h$ switching of quantum conductance associated with a change in nanoscale ferromagnetic domain structure," Applied Physics Letters, vol. 75, no. 11, pp. 1622-1624, 1999.

[18] F. Komori and K. Nakatsuji, "Quantized conductance through atomic-sized iron contacts at $4.2 \mathrm{~K}$," Journal of the Physical Society of Japan, vol. 68, no. 12, pp. 3786-3789, 1999.

[19] N. García, M. Muñoz, and Y.-W. Zhao, "Magnetoresistance in excess of $200 \%$ in ballistic $\mathrm{Ni}$ nanocontacts at room temperature and 100 Oe," Physical Review Letters, vol. 82, no. 14 , pp. 2923-2926, 1999.

[20] H. D. Chopra and S. Z. Hua, "Ballistic magnetoresistance over $3000 \%$ in Ni nanocontacts at room temperature," Physical Review B, vol. 66, no. 2, Article ID 020403, 3 pages, 2002.

[21] A. I. Yanson, "Atomic chains and electronic shells: quantum mechanisns for the formation of nanowires," $\mathrm{Ph}$. D. thesis, Universiteit Leiden, Leiden, The Netherlands, 2001.

[22] D. J. Bakker, Y. Noat, A. I. Yanson, and J. M. van Ruitenbeek, "Effect of disorder on the conductance of a $\mathrm{Cu}$ atomic point contact," Physical Review B, vol. 65, no. 23, Article ID 235416, 5 pages, 2002.

[23] J. Li, T. Kanzaki, K. Murakoshi, and Y. Nakato, "Metaldependent conductance quantization of nanocontacts in solution," Applied Physics Letters, vol. 81, no. 1, pp. 123-125, 2002.

[24] R. H. M. Smit, "From quantum point contacts to monatomic chains: fabrication and characterization of the ultimate nanowire," Ph. D. thesis, Universiteit Leiden, Leiden, The Netherlands, 2003.

[25] V. Rodrigues, J. Bettini, P. C. Silva, and D. Ugarte, "Evidence for spontaneous spin-polarized transport in magnetic nanowires," Physical Review Letters, vol. 91, no. 9, Article ID 096801, 4 pages, 2003.

[26] C. Untiedt, D. M. T. Dekker, D. Djukic, and J. M. van Ruitenbeek, "Absence of magnetically induced fractional quantization in atomic contacts," Physical Review B, vol. 69, no. 8, Article ID 081401, 4 pages, 2004.

[27] C.-S. Yang, C. Zhang, J. Redepenning, and B. Doudin, “In situ magnetoresistance of Ni nanocontacts," Applied Physics Letters, vol. 84, no. 15, pp. 2865-2867, 2004.

[28] M. R. Sullivan, D. A. Boehm, D. A. Ateya, S. Z. Hua, and H. D. Chopra, "Ballistic magnetoresistance in nickel singleatom conductors without magnetostriction," Physical Review $B$, vol. 71, no. 2, Article ID 024412, 8 pages, 2005.
[29] K. Sekiguchi, E. Saitoh, and H. Miyajima, "Conductance quantization by the application of magnetic fields in ballistic Ni nanocontacts," Journal of Applied Physics, vol. 97, no. 10, p. 3 pages, 2005.

[30] M. Díaz, J. L. Costa-Krämer, and P. A. Serena, "Partial versus total conductance histograms: a tool to identify magnetic effects in nanocontacs," Journal of Magnetism and Magnetic Materials, vol. 305, no. 2, pp. 497-503, 2006.

[31] D. Jacob, M. J. Caturla, M. R. Calvo, C. Untiedt, and J. J. Palacios, "Mechanical and electrical properties of $\mathrm{Ni}$ nanocontacts," in Proceedings of IEEE Nanotechnology Materials and Devices Conference (NMDC '06), vol. 1, pp. 236-237, Gyeongju, South Korea, October 2006.

[32] C. Untiedt, M. J. Caturla, M. R. Calvo, J. J. Palacios, R. C. Segers, and J. M. van Ruitenbeek, "Formation of a metallic contact: jump to contact revisited," Physical Review Letters, vol. 98, no. 20, Article ID 206801, 4 pages, 2007.

[33] D. Jacob, J. Fernández-Rossier, and J. J. Palacios, "Magnetic and orbital blocking in Ni nanocontacts," Physical Review B, vol. 71, no. 22, Article ID 220403, 4 pages, 2005.

[34] M. Díaz, J. L. Costa-Krämer, P. A. Serena, E. Medina, and A. Hasmy, "Simulations and experiments of aluminum conductance histograms," Nanotechnology, vol. 12, no. 2, pp. 118-120, 2001.

[35] E. Medina, M. Díaz, N. León, et al., "Ionic shell and subshell structures in aluminum and gold nanocontacts," Physical Review Letters, vol. 91, no. 2, Article ID 026802, 4 pages, 2003.

[36] A. Hasmy, A. J. Pérez-Jiménez, J. J. Palacios, et al., "Ballistic resistivity in aluminum nanocontacts," Physical Review B, vol. 72, no. 24, Article ID 245405, 5 pages, 2005.

[37] P. García-Mochales, S. Peláez, P. A. Serena, E. Medina, and A. Hasmy, "Breaking processes in nickel nanocontacts: a statistical description," Applied Physics A, vol. 81, no. 8, pp. 1545-1549, 2005.

[38] F. Pauly, M. Dreher, J. K. Viljas, M. Häfner, J. C. Cuevas, and P. Nielaba, "Theoretical analysis of the conductance histograms and structural properties of $\mathrm{Ag}, \mathrm{Pt}$, and Ni nanocontacts," Physical Review B, vol. 74, no. 23, Article ID 235106, 21 pages, 2006.

[39] U. Landman, W. D. Luedtke, N. A. Burnham, and R. J. Colton, "Atomistic mechanisms and dynamics of adhesion, nanoindentation, and fracture," Science, vol. 248, no. 4954, pp. 454-461, 1990.

[40] W. D. Luedtke and U. Landman, "Solid and liquid junctions," Computational Materials Science, vol. 1, no. 1, pp. 1-24, 1992.

[41] A. M. Bratkovsky, A. P. Sutton, and T. N. Todorov, "Conditions for conductance quantization in realistic models of atomicscale metallic contacts," Physical Review B, vol. 52, no. 7, pp. 5036-5051, 1995.

[42] M. R. Sørensen, M. Brandbyge, and K. W. Jacobsen, "Mechanical deformation of atomic-scale metallic contacts: structure and mechanisms," Physical Review B, vol. 57, no. 6, pp. 32833294, 1998.

[43] H. Ikeda, Y. Qi, T. Çagin, K. Samwer, W. L. Johnson, and W. A. Goddard III, "Strain rate induced amorphization in metallic nanowires," Physical Review Letters, vol. 82, no. 14, pp. 29002903, 1999.

[44] P. S. Branício and J.-P. Rino, "Large deformation and amorphization of Ni nanowires under uniaxial strain: a molecular dynamics study," Physical Review B, vol. 62, no. 24, pp. 16950$16955,2000$.

[45] S. R. Bahn and K. W. Jacobsen, "Chain formation of metal atoms," Physical Review Letters, vol. 87, no. 26, Article ID 266101, 4 pages, 2001. 
[46] J. W. T. Heemskerk, Y. Noat, D. J. Bakker, J. M. van Ruitenbeek, B. J. Thijsse, and P. Klaver, "Current-induced transition in atomic-sized contacts of metallic alloys," Physical Review B, vol. 67, no. 11, Article ID 115416, 5 pages, 2003.

[47] Y.-H. Wen, Z.-Z. Zhu, G.-F. Shao, and R.-Z. Zhu, "The uniaxial tensile deformation of $\mathrm{Ni}$ nanowire: atomic-scale computer simulations," Physica E, vol. 27, no. 1-2, pp. 113120, 2005.

[48] B. Wang, D. Shi, J. Jia, G. Wang, X. Chen, and J. Zhao, "Elastic and plastic deformations of nickel nanowires under uniaxial compression," Physica E, vol. 30, no. 1-2, pp. 45-50, 2005.

[49] H. S. Park, K. Gall, and J. A. Zimmerman, "Shape memory and pseudoelasticity in metal nanowires," Physical Review Letters, vol. 95, no. 25, Article ID 255504, 4 pages, 2005.

[50] M. Dreher, F. Pauly, J. Heurich, J. C. Cuevas, E. Scheer, and P. Nielaba, "Structure and conductance histogram of atomicsized Au contacts," Physical Review B, vol. 72, no. 7, Article ID 075435, 11 pages, 2005.

[51] M. S. Daw and M. I. Baskes, "Semiempirical, quantum mechanical calculation of hydrogen embrittlement in metals," Physical Review Letters, vol. 50, no. 17, pp. 1285-1288, 1983.

[52] S. M. Foiles, "Application of the embedded-atom method to liquid transition metals," Physical Review B, vol. 32, no. 6, pp. 3409-3415, 1985.

[53] Y. Mishin, D. Farkas, M. J. Mehl, and D. A. Papaconstantopoulos, "Interatomic potentials for monoatomic metals from experimental data and ab initio calculations," Physical Review $B$, vol. 59, no. 5, pp. 3393-3407, 1999.

[54] W. Liang and M. Zhou, "Atomistic simulations reveal shape memory of fcc metal nanowires," Physical Review B, vol. 73, no. 11, Article ID 115409, 11 pages, 2006.

[55] Y. V. Sharvin, "A possible method for studying fermi surfaces," Zhurnal Eksperimental'noi $i$ Teoreticheskoi Fiziki, vol. 48, pp. 984-985, 1965.

[56] Y. V. Sharvin, "A possible method for studying fermi surfaces," Soviet Physics JETP, vol. 21, pp. 655-656, 1965.

[57] H. Mehrez and S. Ciraci, "Yielding and fracture mechanisms of nanowires," Physical Review B, vol. 56, no. 19, pp. 12632$12642,1997$.

[58] P. Sen, O. Gülseren, T. Yildirim, I. P. Batra, and S. Ciraci, "Pentagonal nanowires: a first-principles study of the atomic and electronic structure," Physical Review B, vol. 65, no. 23, Article ID 235433, 7 pages, 2002.

[59] J. C. Gonzälez, V. Rodrigues, J. Bettini, et al., "Indication of unusual pentagonal structures in atomic-size $\mathrm{Cu}$ namwires," Physical Review Letters, vol. 93, no. 12, Article ID 126103, 4 pages, 2004.

[60] O. Gülseren, F. Ercolessi, and E. Tosatti, "Noncrystalline structures of ultrathin unsupported nanowires," Physical Review Letters, vol. 80, no. 17, pp. 3775-3778, 1998.

[61] Q. Pu, Y. Leng, L. Tsetseris, H. S. Park, S. T. Pantelides, and P. T. Cummings, "Molecular dynamics simulations of stretched gold nanowires: the relative utility of different semiempirical potentials," Journal of Chemical Physics, vol. 126, no. 14, Article ID 144707, 6 pages, 2007. 

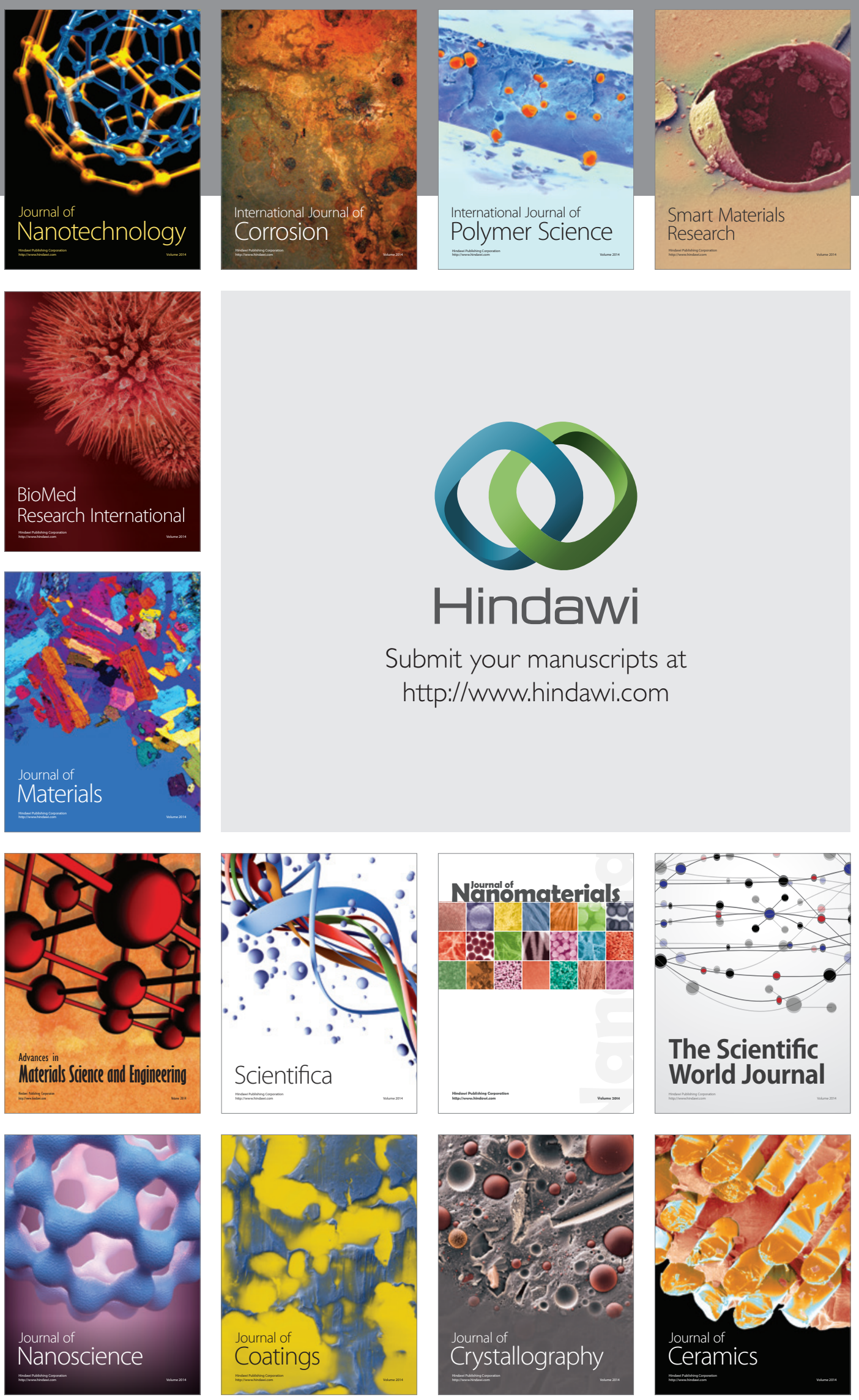

The Scientific World Journal

Submit your manuscripts at

http://www.hindawi.com

\section{World Journal}

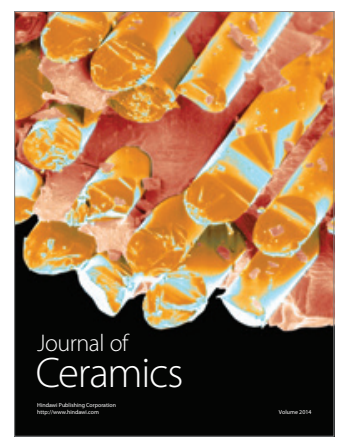

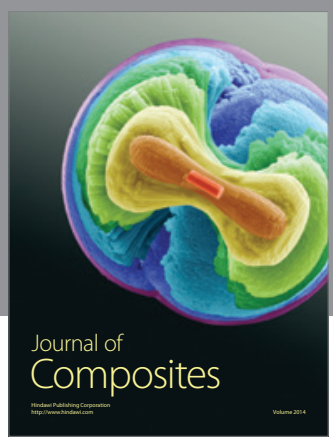
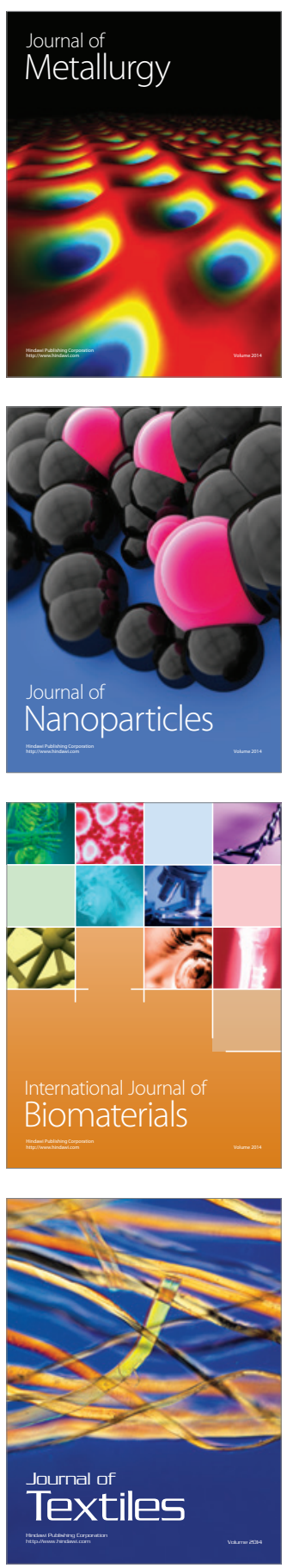